Supporting Information

for

\title{
"Realizing Vibrant and High-Contrast Reflective Structural Colors from Lossy Metals Supporting Dielectric Gratings"
}

Youngji Kim, ${ }^{\dagger}$ Kyungmin Jung, ${ }^{\dagger}$ Jiung Cho, ${ }^{\ddagger}$ and Jerome K. Hyun ${ }^{* \dagger}$

${ }^{\dagger}$ Department of Chemistry and Nanoscience, Ewha Womans University, Seoul 03760, Republic of Korea

₹Western Seoul center, Korea Basic Science Institute, Seoul 03760, Republic of Korea

*E-mail: kadam.hyun@ewha.ac.kr 


\section{Sample fabrication}

a) metal film evaporation $\quad$ b) PMMA spin coating

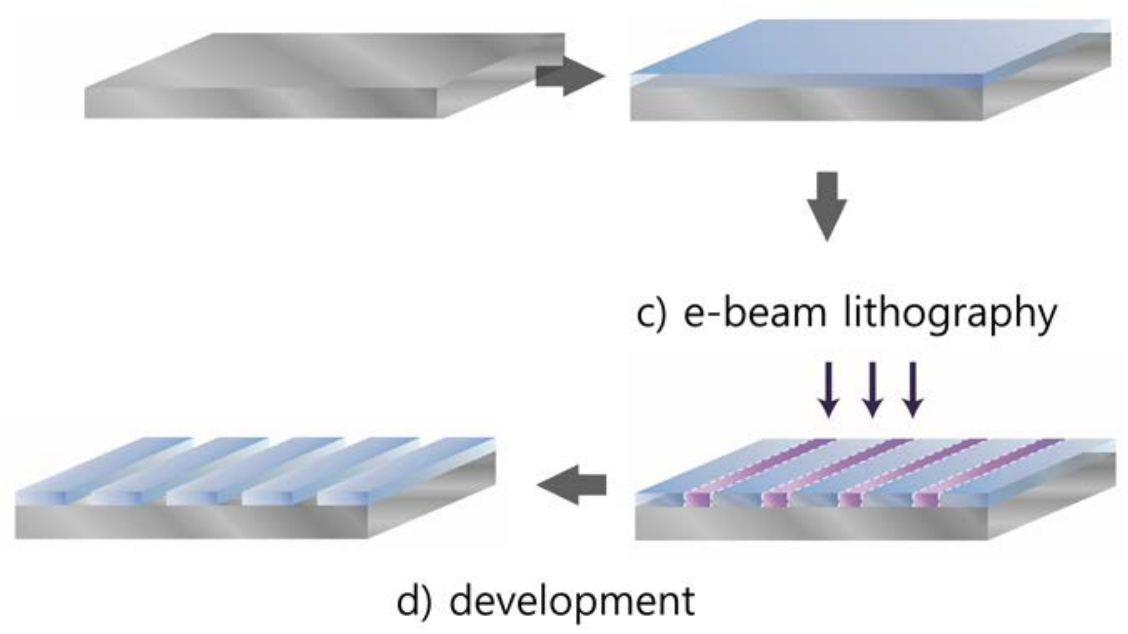

Figure S1. Fabrication process of dielectric grating on metal. a) A metal film (Ag, Pt or W) of optically opaque thickness is deposited onto a Si substrate using e-beam evaporation or DC sputtering. b) A PMMA layer is spin-coated onto the metal substrate. c) Line grating patterns are written onto the PMMA layer by e-beam lithography. d) Sample is developed. 


\section{Measurement setup}

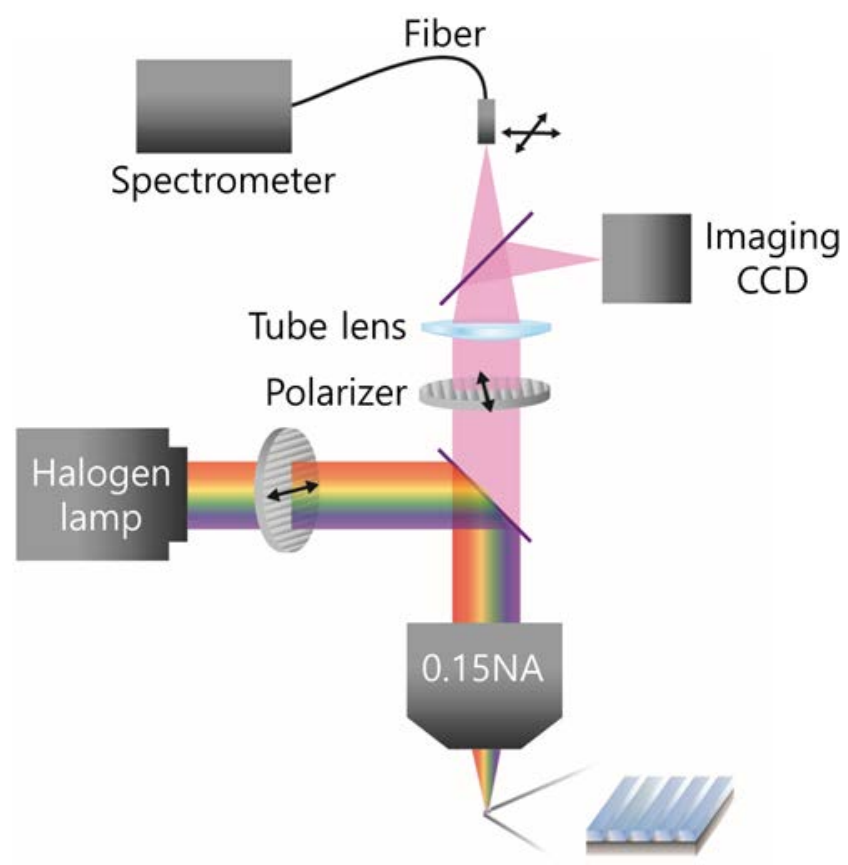

Figure S2. Schematic of the cross-polarized input-output setup. 


\section{Analytical description of the reflection}

Our analytical description of reflection from a 1D dielectric grating on a metal substrate using coupled waveguide-array modes is derived from the work of W. Yang et al. ${ }^{1} \mathrm{~W}$. Yang provides a detailed description of the analytical treatment of coupled waveguide-array modes in a freestanding grating system, where the top, middle and bottom layers are air, grating and air, respectively. Our description modifies the original system by replacing the transmissive bottom dielectric layer with a reflective PEC layer.

We consider a system consisting of an air (layer I), dielectric grating (layer II) and PEC (layer III) layer, as shown in Figure S3. Analogous to the work of W. Yang, we can expand the fields in the air and dielectric grating layers according to their modal basis set as follows.

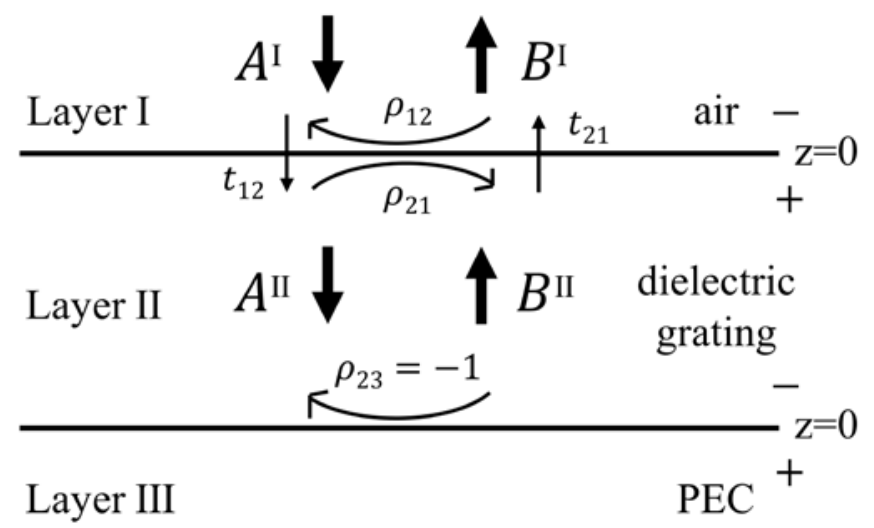

Figure S3. Schematic of mode propagation in air-grating-PEC system

$$
\begin{aligned}
& E_{x}^{I}(x, z)=\sum_{n=-\infty}^{+\infty}\left[\boldsymbol{A}_{n}^{I}(z)+\boldsymbol{B}_{n}^{I}(z)\right] \varepsilon_{x, n}^{I}(x) \\
& E_{x}^{I}(x, z)=\sum_{m=0}^{\infty}\left[\boldsymbol{A}_{\boldsymbol{m}}^{I}(z)+\boldsymbol{B}_{\boldsymbol{m}}^{I I}(z)\right] \varepsilon_{x, m}^{I}(x)
\end{aligned}
$$

Here, the coefficients characterize the complex diffraction and waveguide-array modes in the air and grating layers, respectively, propagating in the forward $(A)$ and backward(B) directions. The forward moving modes can be expressed as follows.

$\boldsymbol{A}^{I}=\left[\begin{array}{llllllll}\cdots & \boldsymbol{A}_{-2}^{I} & \boldsymbol{A}_{-1}^{I} & \boldsymbol{A}_{\mathbf{0}}^{I} & \boldsymbol{A}_{1}^{I} & \boldsymbol{A}_{2}^{I} & \cdots\end{array}\right]^{T}$ 
$A^{I I}=\left[\begin{array}{lll}A_{0}^{I I} & A_{1}^{I I} & A_{2}^{I I}\end{array}\right]^{T}$

The backward-moving modes can also be expressed analogously. $\boldsymbol{\rho}_{21}$ and $\boldsymbol{\rho}_{12}$ are reflection matrices describing the reflection of modes from layer 2 to 1 and 1 to 2, respectively. $\boldsymbol{\rho}_{21}$, in particular, describes the coupling of waveguide-array modes into one another. Similarly, $\boldsymbol{\tau}_{12}$ and $\boldsymbol{\tau}_{21}$ are transmission matrices for modes transmitting from layer 1 to 2 and 2 to 1 , respectively. $\boldsymbol{\rho}_{23}$ describes the waveguide-array modes reflecting off the PEC layer $\left(\boldsymbol{\rho}_{\mathbf{2} 3}=\mathbf{- 1}\right)$ in which case all modes reflect onto themselves (i.e., no cross-coupling among modes occurs). Our objective is to find an expression for the $0^{\text {th }}$ order reflection, which can be obtained by finding

$\mathbf{R}=B_{I} \cdot A_{I}^{-1}$

We can first construct a matching matrix that relates the fields at the interface between air and grating.

$$
\begin{aligned}
& \left(\begin{array}{l}
\boldsymbol{A}_{I}\left(z=0^{-}\right) \\
B_{I}\left(z=0^{-}\right)
\end{array}\right) \\
& =\left(\begin{array}{cc}
\boldsymbol{t}_{\mathbf{1 2}}{ }^{-1} & -\boldsymbol{t}_{\mathbf{1 2}}{ }^{-1} \boldsymbol{\rho}_{\mathbf{2 1}} \\
\boldsymbol{\rho}_{\mathbf{1 2}} \boldsymbol{t}_{\mathbf{1 2}}{ }^{-1} & -\boldsymbol{\rho}_{\mathbf{1 2}} \boldsymbol{t}_{\mathbf{1 2}}{ }^{-1} \boldsymbol{\rho}_{\mathbf{2 1}}+\boldsymbol{t}_{\mathbf{2 1}}
\end{array}\right)\left(\begin{array}{l}
\boldsymbol{A}_{I}\left(z=0^{+}\right) \\
B_{I I}\left(z=0^{+}\right)
\end{array}\right)
\end{aligned}
$$

We define a propagation matrix that tracks the accumulated phase of the modes as they propagate through the grating thickness.

$$
\boldsymbol{\varphi}=\left(\begin{array}{ccc}
e^{-i \beta_{0} h} & 0 & 0 \\
0 & e^{-i \beta_{1} h} & 0 \\
0 & 0 & e^{-i \beta_{2} h}
\end{array}\right)
$$

The modes at the top grating interface can then be related to the bottom interface as follows. 


$$
\begin{aligned}
& \left(\begin{array}{l}
A_{I}\left(z=0^{+}\right) \\
B_{I}\left(z=0^{+}\right)
\end{array}\right) \\
& =\left(\begin{array}{cc}
\varphi^{-1} & 0 \\
0 & \varphi
\end{array}\right)\left(\begin{array}{l}
A_{I I}\left(z=h^{-}\right) \\
B_{I I}\left(z=h^{-}\right)
\end{array}\right)
\end{aligned}
$$

The diffraction modes in the air layer can then be expressed as follows.

$$
\left(\begin{array}{l}
\boldsymbol{A}_{I}\left(z=0^{-}\right) \\
B_{I}\left(z=0^{-}\right)
\end{array}\right)=\left(\begin{array}{cc}
\boldsymbol{t}_{12}{ }^{-1} & -\boldsymbol{t}_{\mathbf{1 2}}^{-1} \boldsymbol{\rho}_{\mathbf{2 1}} \\
\boldsymbol{\rho}_{\mathbf{1 2}} \boldsymbol{t}_{\mathbf{1 2}}{ }^{-1} & -\boldsymbol{\rho}_{\mathbf{1 2}} \boldsymbol{t}_{\mathbf{1 2}}^{-1} \boldsymbol{\rho}_{\mathbf{2 1}}+\boldsymbol{t}_{\mathbf{2 1}}
\end{array}\right)\left(\begin{array}{cc}
\boldsymbol{\varphi}^{-1} & 0 \\
0 & \varphi
\end{array}\right)\left(\begin{array}{c}
\boldsymbol{A}_{I}\left(z=h^{-}\right) \\
B_{I}\left(z=h^{-}\right)
\end{array}\right)
$$

Since $\mathbf{B}_{\text {II }}$ are AII modes reflecting from a PEC layer, they can be expressed as

$$
\boldsymbol{B}_{I I}\left(z=h^{-}\right)=\boldsymbol{\rho}_{23} \boldsymbol{A}_{I}\left(z=h^{-}\right)=-\boldsymbol{A}_{I}\left(z=h^{-}\right)
$$

Using equation (3), (7) and (8), we can find an expression for the reflection as follows

$$
\mathrm{R}=\left(\rho_{12} t_{12}^{-1} \varphi^{-1}+\rho_{12} t_{12}^{-1} \rho_{21} \varphi-t_{21} \varphi\right)\left(\mathrm{t}_{12}^{-1} \varphi^{-1}+\mathrm{t}_{12}{ }^{-1} \rho_{21} \varphi\right)^{-1},
$$

from which we find R00. Expressions for $\boldsymbol{\rho}_{\mathbf{1 2}}, \boldsymbol{\rho}_{\mathbf{2 1}}, \boldsymbol{t}_{\mathbf{1 2}}$ and $\boldsymbol{t}_{\mathbf{2 1}}$ can be found from below ${ }^{1}$

$\boldsymbol{\rho}_{21}=(\mathbf{H}+\mathbf{E})^{-1}(\mathbf{H}-\mathbf{E})$

$\boldsymbol{\rho}_{\mathbf{1 2}}=-\boldsymbol{H}(\mathbf{H}+\mathbf{E})^{-1}(\mathbf{H}-\mathbf{E}) \boldsymbol{H}^{-1}$

$\boldsymbol{t}_{\mathbf{2 1}}=2 \boldsymbol{H}(\mathbf{H}+\mathbf{E})^{-1} \mathbf{E}$

$\boldsymbol{t}_{12}=2(\mathbf{H}+\mathbf{E})^{-1}$,

where $\mathbf{H}$ and $\mathbf{E}$ correspond to the overlap of fields in air and grating, expressed as,

$$
\begin{aligned}
\boldsymbol{H}_{\boldsymbol{n}, \boldsymbol{m}} & =\frac{\int_{0}^{\wedge} H_{y, m}^{I}(x)\left[H_{y, n}^{I}(x)\right]^{*} d x}{\int_{0}^{\wedge}\left|H_{y, n}^{I}(x)\right|^{2} d x} \\
\boldsymbol{E}_{\boldsymbol{n}, \boldsymbol{m}} & =\frac{\int_{0}^{\wedge} \varepsilon_{x, m}^{I}(x)\left[\varepsilon_{x, n}^{I}(x)\right]^{*} d x}{\int_{0}^{\wedge}\left|\varepsilon_{x, n}^{I}(x)\right|^{2} d x} .
\end{aligned}
$$



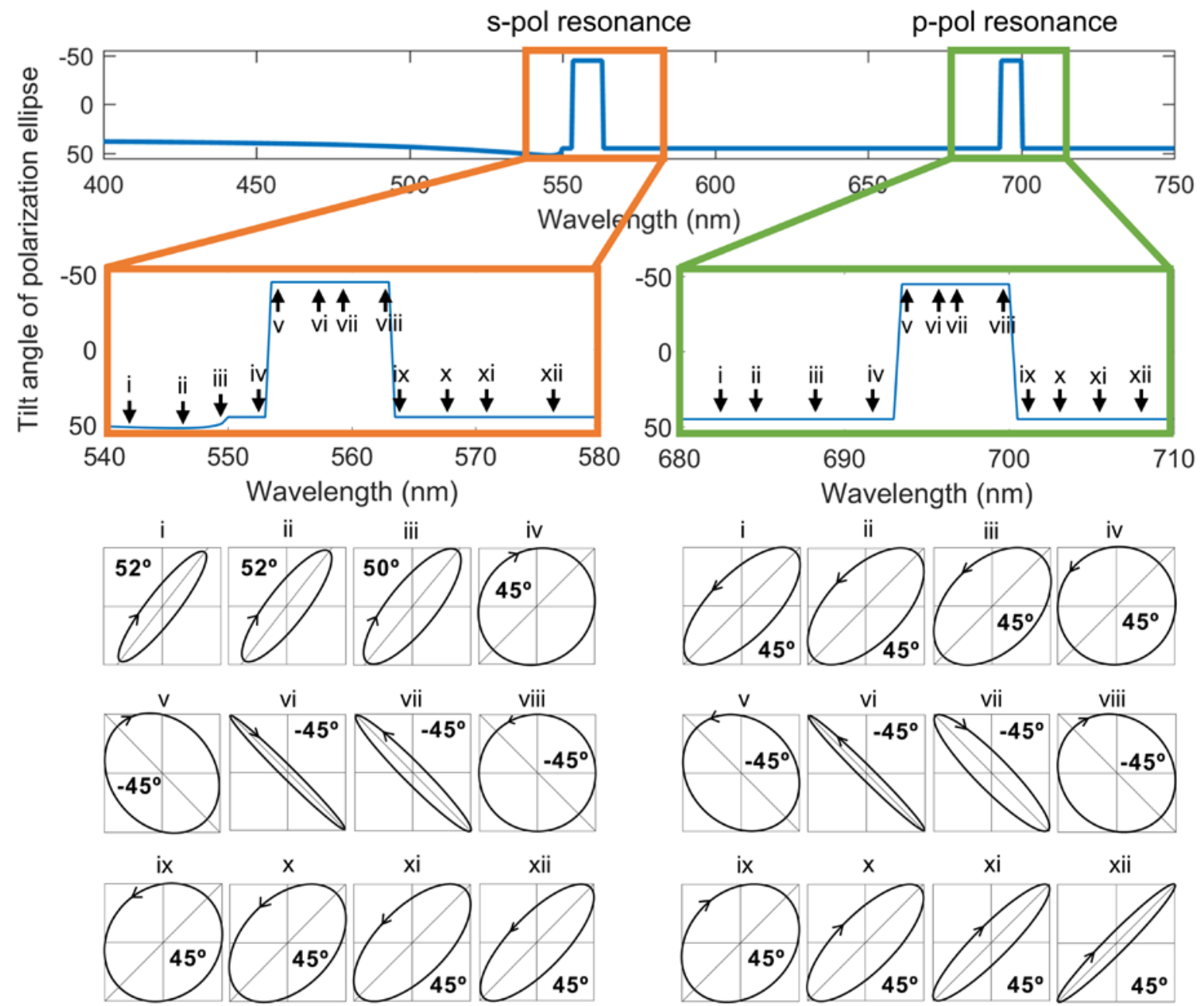

Figure S4. Tilt angle of polarization ellipse from Figure 2g denoted by an expanded number of polarization ellipses at the s-pol and p-pol resonance. 
a

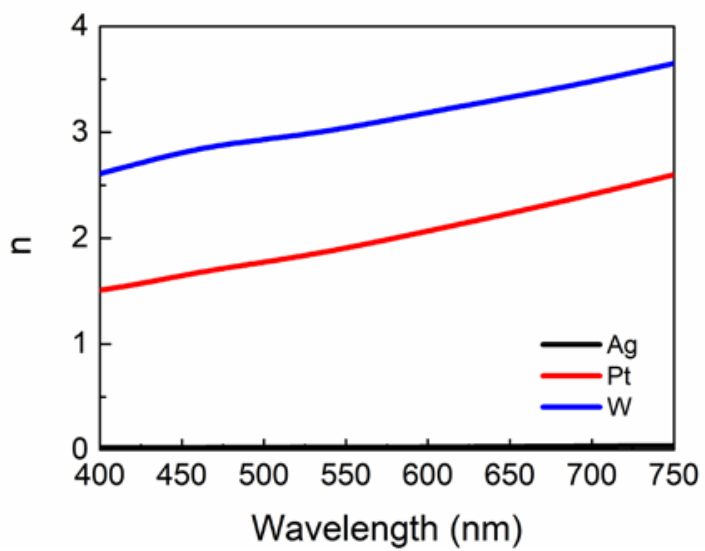

b

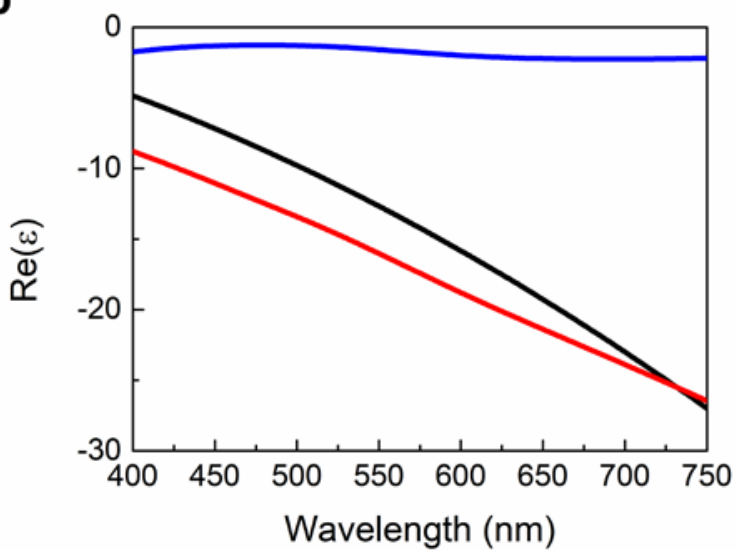

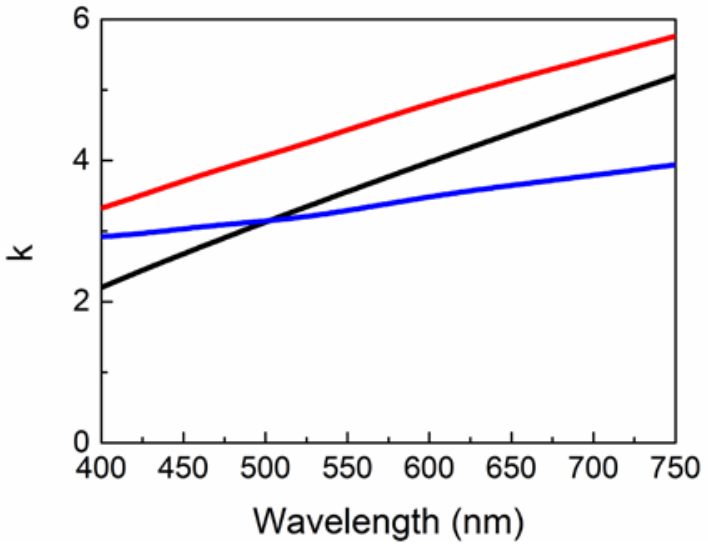

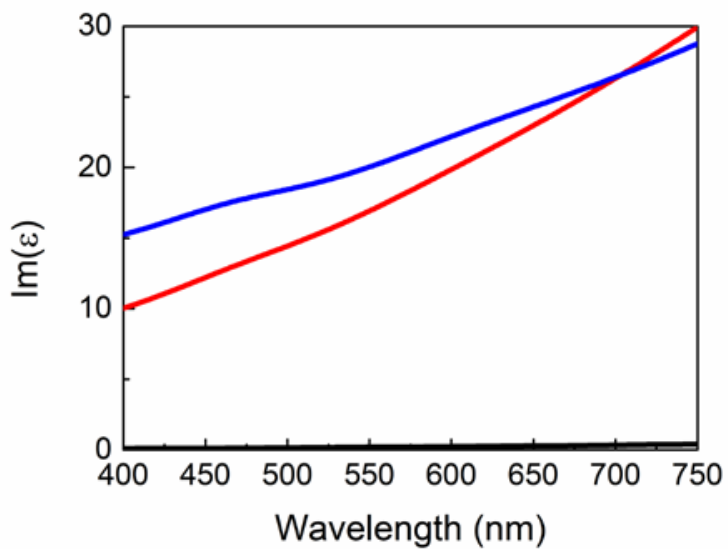

Figure S5. (a) Refractive indices, and (b) real and imaginary permittivities of Ag, Pt and W. 
a

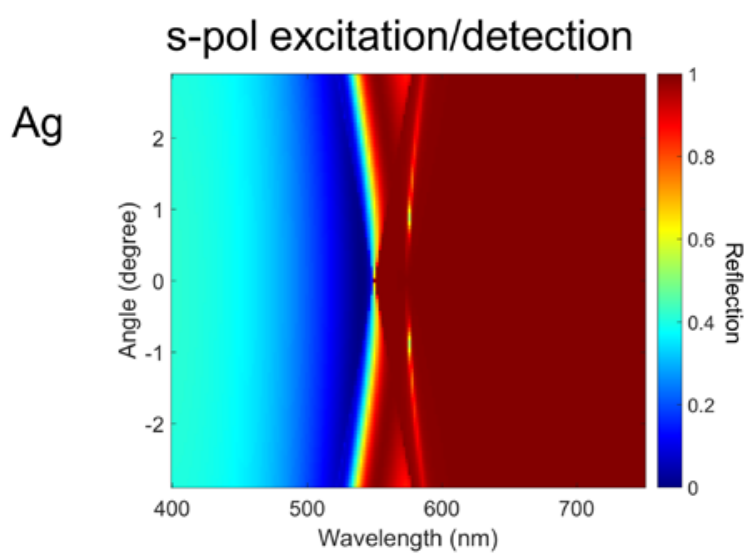

$\mathrm{Pt}$

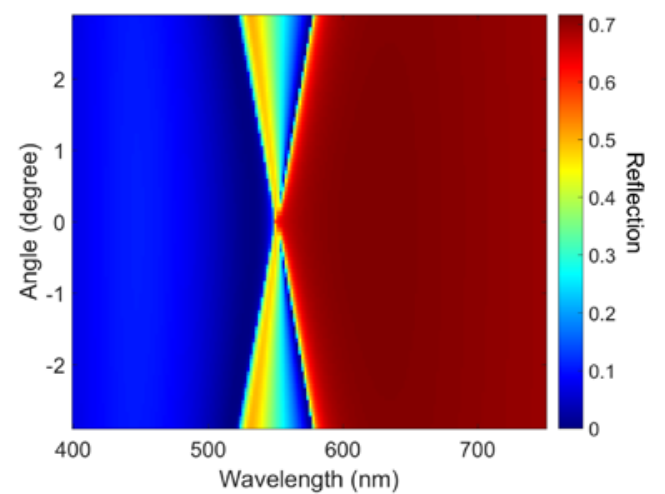

W

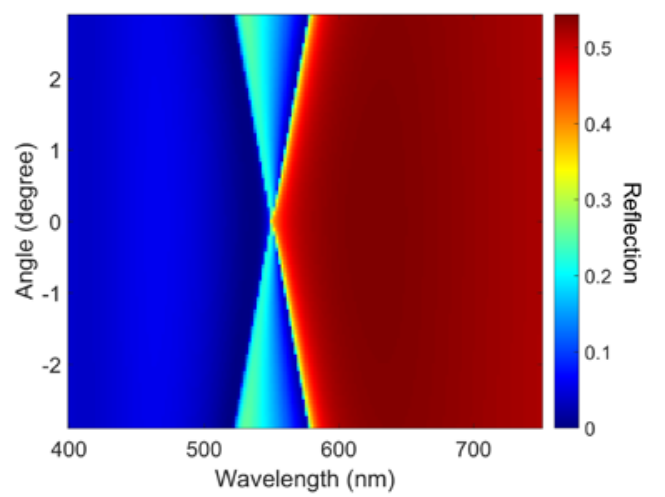

b
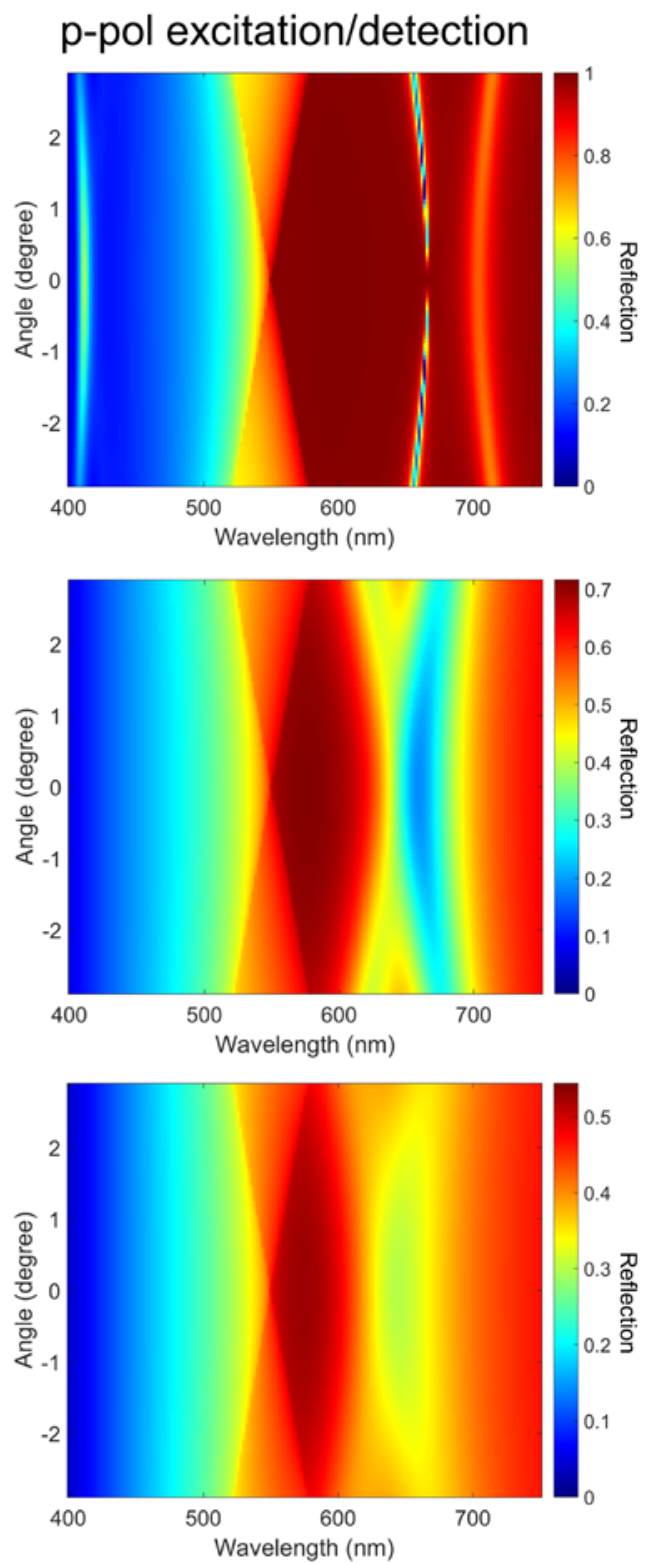

Figure S6. Calculated (a) s-pol and (b) p-pol $0^{\text {th }}$ order reflection from PMMA grating on Ag (top), Pt (middle) and W (bottom) substrates as a function of wavelength and incident angle. Grating parameters are identical to those used in Figure 4. 
a

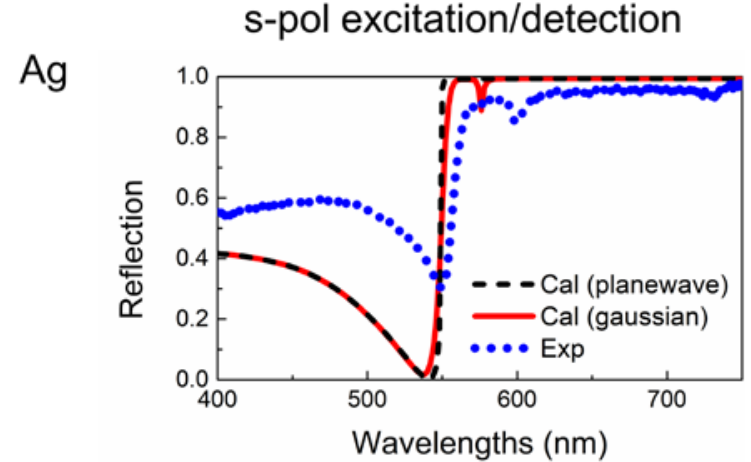

Pt

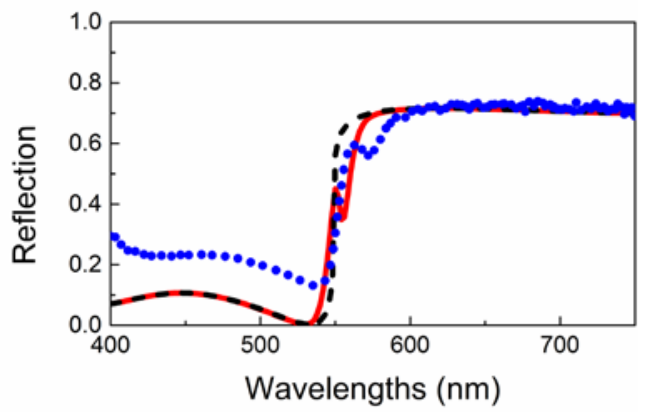

W

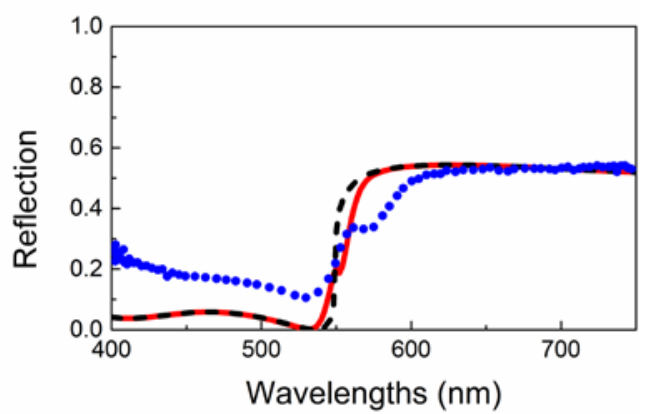

b p-pol excitation/detection
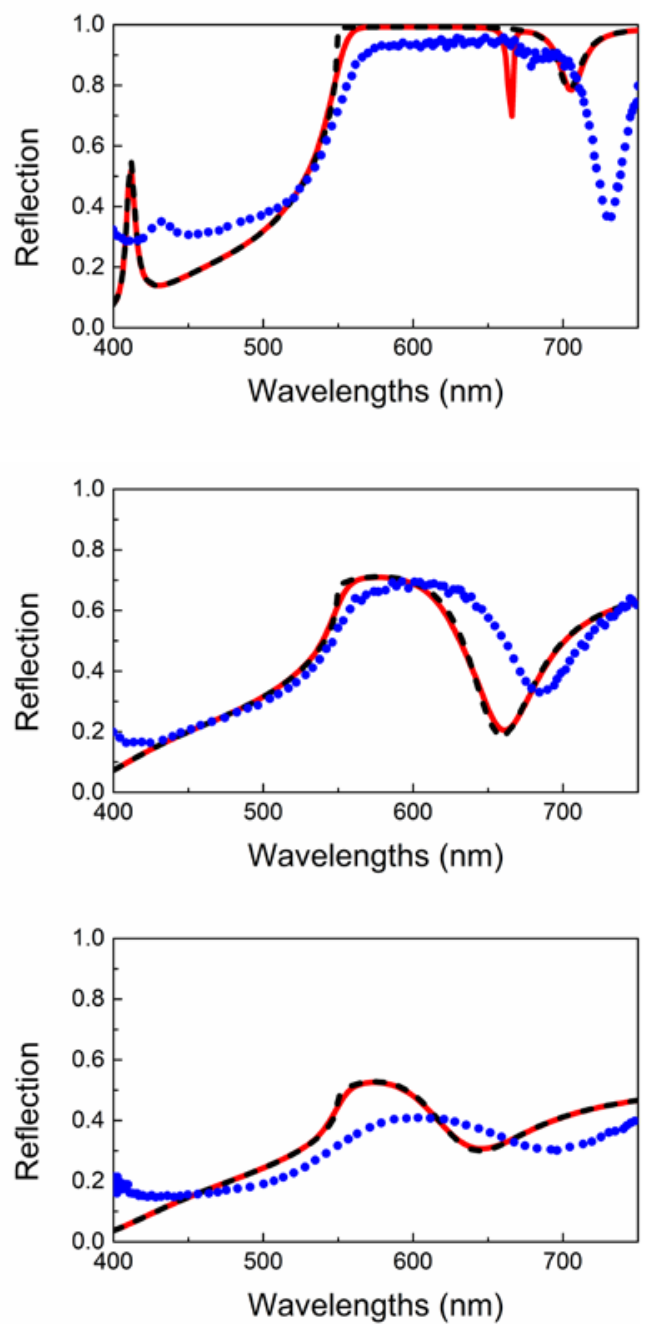

Figure S7. Comparison of (a) s-pol and (b) p-pol $0^{\text {th }}$ order reflection between measurement, and calculation using a planewave source and Gaussian beam for Ag (top), Pt (middle), and W (bottom) substrates. Reflection from a planewave source corresponds to the spectrum at the $0^{\circ}$ angle in Figure S6, while reflection from a Gaussian beam corresponds to the product between the angular Gaussian distribution of incident light and the incident angle dependent reflection (Figure S6) integrated over the angles. Grating parameters are identical to those used in Figure 4. 


\section{Dependence of propagation length on grating parameters}

To establish general trends in loss for different grating parameters, we can, to a first approximation, characterize the grating with an effective refractive index in the subwavelength regime, and consider the expression of an SPP wavevector for a flat dielectric-metal interface, $\beta=2 \pi / \lambda \sqrt{\epsilon_{m} \epsilon_{d} /\left(\epsilon_{m}+\epsilon_{d}\right)}$, where $\epsilon_{m}$ and $\epsilon_{d}$ describe the metal and effective dielectric permittivities, respectively. A similar approximation was also implemented to approximate the dispersion of SPPs in a metallic slab with a hole array. ${ }^{2}$ We note, however, in our case that the effective dielectric permittivity is a function of period.

A useful measure of SPP loss is the propagation length which depends inversely on the imaginary part of the SPP wavevector: $L_{S P P}=1 /(2 \operatorname{Im}(\beta))$. An increase in the duty cycle (DC), or grating refractive index has the effect of increasing the effective refractive index of the grating, or equivalently $\epsilon_{d}$, which increases the imaginary part of the wavevector and consequently decreases the propagation length. Because the gratings exhibit a fixed etch width, an increase in period results in an increase in duty cycle, increasing dissipation manifested by a decreased SPP propagation length for Pt and W substrates as shown in Figure 4d.

For Ag, the propagation length increases by several folds over the span of wavelengths which offsets the increase in dissipation due to the larger effective index (See Figure S8). Figure S8 shows the calculated propagation length of SPPs from dielectrics with an effective index of 1.37, 139, and 1.41 on Ag, Pt and W. These indices were extracted from the $0^{\text {th }}$ order waveguide-array wavenumber for gratings with a period of 400, 550 and 700, etch width of 150 and index of 1.5 to approximate the effective index at different grating parameters. Despite ignoring that the SPP wavevector depends on the period, which is apparent in the dispersion relation of our model and the use of a fixed effective permittivity over wavelengths, we can see that the propagation length is an order of magnitude higher than that of SPPs on Pt and W. 
Accordingly, the dissipation for SPPs on Ag does not increase for larger periods as seen in Figure 4d. It is important to note that this approximation does not capture the exact nature of the dissipation, yet provides simple and general guidelines for optimizing dissipation with the grating parameters.

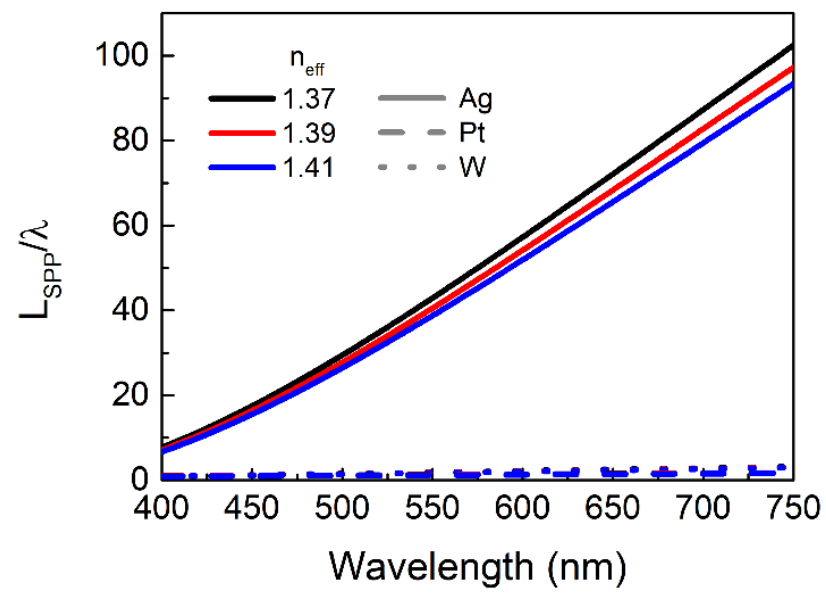

Figure S8. SPP propagation length normalized by the wavelength, calculated for a smooth Ag (solid line), Pt (dashed line) and W (dotted line) film supporting dielectrics of different effective refractive indices. 


\section{Reference}

1 Chang-Hasnain, C. J.; Yang, W. High-Contrast Gratings for Integrated Optoelectronics. Adv. Opt. Photonics 2012, 4, 379-440

2 Ghaemi, H. F.; Thio, T.; Grupp, D. E.; Ebbesen, T. W.; Lezec, H. J. Surface

Plasmons Enhance Optical Transmission through Subwavelength Holes. Phys. Rev. B 1998, $58,6779-6782$ 